\title{
Diskursivität und Einheit des Bewusstseins bei Kant
}

\author{
Dietmar H. Heidemann (Universität Luxemburg)
}

\section{Einleitung}

Für eine philosophische Theorie des Bewusstseins ist der Begriff der Einheit unentbehrlich. Denn ohne den Begriff der Einheit dürfte nur schwer zu erfassen sein, was Bewusstsein grundsätzlich auszeichnet, worin seine Funktion besteht und wie kognitive Leistungen des Bewusstseins zustande kommen. Dieser Befund zählt zu den Grundeinsichten auch der Kantischen Theorie des Bewusstseins und Selbstbewusstseins. Dabei ist Kant wahrscheinlich der erste Philosoph, der jenseits des Lehrkanons klassischer Ontologien eine detailliert ausgearbeitete Theorie des Bewusstseins und seiner rein kognitiven Fähigkeiten vorgelegt hat. Diese Theorie zeichnet sich entschieden durch den Anspruch aus, keine ontologischen Annahmen hinsichtlich des Bewusstseins und seines Inhabers $\mathrm{zu}$ machen, so dass mit dem Ausdruck ,Einheit des Bewusstseins' insbesondere nicht die mentale Einheit einer existierenden Geistsubstanz gemeint ist, sondern anderes bezeichnet sein muss. ${ }^{1}$

Dass ,Einheit' ein Schlüsselbegriff philosophischer Theoriebildung über Bewusstsein ist, stellt für Kant durchaus keine Selbstverständlichkeit oder begriffliche Plattitüde dar. ,Einheit' als Eigenschaft menschlichen Bewusstseins ist nach Kant ein kontingentes kognitives Merkmal des Bewusstseins, da wir uns leicht eine Welt vorstellen können, in der Bewusstsein nicht durch ,Einheit' geprägt ist, zumindest nicht durch diejenige ,Einheit' wie sie unserem Bewusstsein in unserer Welt offensichtlich zukommt. Dies zeigt nicht zuletzt die Idee des intuitiven Verstandes, wie sie Kant in $\S 77$ der Kritik der Urteilskraft rein hypothetisch und negativkontrastierend zum menschlichen Verstand konzipiert. Gegenüber dem intuitiven Verstand ist menschliches Bewusstsein und damit der menschliche diskursive Verstand ein bloß zufälliges Vermögen. Obwohl der intuitive Verstand ein nichtdiskursives kognitives Vermögen darstellt, kommt auch er nicht ohne den Begriff der Einheit aus, eine intuitive mentale Einheit jedoch, die mit der diskursiven Einheit menschlichen Bewusstseins nur den Namen gemein hat. Schließlich besteht die

\footnotetext{
${ }^{1}$ Im Folgenden werden die Begriffe ,Bewusstsein' und ,Selbstbewusstsein' synonym verwendet. Dort, wo es auf Bedeutungsunterschiede ankommt, wird dies kenntlich gemacht. Eine Definition dieser Begriffe soll an dieser Stelle jedoch nicht gegeben werden. Die spezifisch Kantische Verwendung der Begriffe ,Bewusstsein' und ,Selbstbewusstsein' wird im weiteren aber deutlich werden.
} 
Einheit der Erkenntnis des intuitiven Verstandes in einem nicht-inferentiellen, unmittelbaren Bewussthaben von Objekten, während ein diskursives Vermögen kognitive Einheit mit Hilfe begrifflicher Inferenzen herstellen muss:

„Unser Verstand ist ein Vermögen der Begriffe, d.i. ein discursiver Verstand, für den es freilich zufällig sein muß, welcherlei und wie sehr verschieden das Besondere sein mag, das ihm in der Natur gegeben werden und das unter seine Begriffe gebracht werden kann. [...] so kann man sich auch einen intuitiven Verstand (negativ, nämlich bloß als nicht discursiven) denken, welcher nicht vom Allgemeinen zum Besonderen und so zum Einzelnen (durch Begriffe) geht, und für welchen jene Zufälligkeit der Zusammenstimmung der Natur in ihren Producten nach besondern Gesetzen zum Verstande nicht angetroffen wird, welche dem unsrigen es so schwer macht, das Mannigfaltige derselben Übereinstimmung der Naturmerkmale zu unserm Vermögen der Begriffe, welche sehr zufällig ist, zu Stande bringen kann, dessen ein anschauender Verstand aber nicht bedarf.“ $(K U 5: 406){ }^{2}$

Auch wenn Kant in der Kritik der reinen Vernunft von der „Einheit des Selbstbewusstseins“ als der „ursprünglich-synthetischen Einheit der Apperzeption“ spricht, die sich auf kein grundlegenderes Prinzip zurückführen lasse (KRV B 131f.) und sich als originäre Eigenschaft menschlichen Bewusstseins weiterer theoretischer Explikation zu entziehen scheint, ist es immer noch eine sinnvolle Frage, was Einheit $\mathrm{zu}$ einer ursprünglichen Eigenschaft menschlichen Bewusstseins macht. Die Unhintergehbarkeit der Einheit des Bewusstseins wird damit nicht bestritten, nicht zuletzt weil nach Kant alle Erkenntnis auf dieser ursprünglichen Einheit beruht, von der wir nicht sagen können, woher sie stammt. Gleichwohl, und dafür wird im

\footnotetext{
${ }^{2}$ Vgl. $K U$ 5: 406: „Es kommt hier also auf das Verhalten unseres Verstandes zur Urtheilskraft an, daß wir nämlich darin eine gewisse Zufälligkeit der Beschaffenheit des unsrigen aufsuchen, um diese Eigenthümlichkeit unseres Verstandes zum Unterschiede von anderen möglichen anzumerken. Diese Zufälligkeit findet sich ganz natürlich in dem Besondern, welches die Urtheilskraft unter das Allgemeine der Verstandesbegriffe bringen soll“. Mit Ausnahme der Kritik der reinen Vernunft werden Kants Werke mit Angabe der Band- und Seitenzahlen zitiert nach: Kants Gesammelte Schriften, Akademie der Wissenschaften (vormals: Preußische Akademie der Wissenschaften), Berlin: Walter de Gruyter 1900ff. Die „Kritik der reinen Vernunft" wird zitiert nach der Ausgabe von Jens Timmermann (Hrsg.), Hamburg 1998 (A für die erster Auflage, B für die zweite Auflage).
} 
Folgenden argumentiert, lässt sich zeigen, warum ,Einheit' eine kognitive Eigenschaft menschlichen Bewusstseins sein muss, auch wenn das Faktum der Einheit des Bewusstseins als solches für den Menschen ein kontingentes ist.

Zeigen lässt sich dies anhand des zweiten Schlüsselbegriffs der Kantischen Theorie des Bewusstseins, des Begriffs der Identität. Wie wir sehen werden, ist der Begriff der Identität des Bewusstseins ebenso wie der Begriff der Einheit nach Kant rein logisch und nicht ontologisch, etwa im Sinne zeitlicher Beharrlichkeit perdurierender Objekte, zu verstehen. Logische Identität, so wie sie von Kant in der transzendentalen Deduktion der Kritik der reinen Vernunft eingeführt wird, ist diskursive Identität wie sie dem Begriff als repraesentatio discursiva zugrundliegt. Auf ihr beruhen die Handlungen des Verstandes qua Apperzeption, der gemäß diskursiven Regeln, das heißt Funktionen, logische Ordnung unter mannigfaltigen Vorstellungen herstellt. Die kognitive Tatsache der Mannigfaltigkeit der Vorstellungen erklärt dabei, aus welchen Gründen menschlichem Bewusstsein neben Einheit ursprünglich auch logisch-diskursive Identität zukommen muss. Denn die für das Bewusstsein mannigfaltigen Vorstellungen müssen synthetisiert werden, um als Begriffe in Urteilen zur objektiven Einheit der Apperzeption gebracht und damit Erkenntnis werden zu können.

Im ersten Abschnitt der folgenden Darlegungen soll dieser abstrakte Sachverhalt anhand des Zusammenhanges von Kantischer Begriffslehre und Theorie des transzendentalen Selbstbewusstseins transparent gemacht werden. Die Einheit des Bewusstseins, so wird die Rekonstruktion dieses Zusammenhanges ergeben, gründet in der Theorie des diskursiven Begriffs. Die dabei herausgearbeitete Konzeption der diskursiven Identität wird dann im zweiten Abschnitt anhand des dritten Paralogismus der ersten Auflage der Kritik der reinen Vernunft mit dem Begriff der personalen Identität kontrastiert, um zu zeigen, warum der auch hier bestehende konstitutive Zusammenhang zwischen kognitiver Einheit und Identität des Bewusstseins zu keiner spezifischen Erkenntnis hinsichtlich des Bewusstseins führt. Während die transzendentale Deduktion die Theorie nichtzeitlicher, logischer Einheit und diskursiver Identität der transzendentalen Apperzeption entfaltet, thematisiert der dritte Paralogismus das mentale Phänomen kontingenter, temporaler Einheit und damit personaler Identität. Über die prinzipiellen Bedeutungsunterschiede dieser beiden Konzeptionen von Einheit und Identität im Bereich des Mentalen ist Klarheit zu schaffen. Der Schlussteil widmet sich einigen naheliegenden Einwänden gegen den 
in diesem Beitrag entwickelten Interpretationsvorschlag. Insbesondere wird eine Kritik an der hier verteidigten Diskursivitätsthese diskutiert, derzufolge die Einheit des Bewusstseins in der Diskursivität des Verstandes bzw. Begriffs und nicht umgekehrt die Diskursivität des Verstandes bzw. des Begriffs in der Einheit des Bewusstseins gründet.

\section{Logische Einheit und diskursive Identität des Bewusstseins}

Es ist zunächst nicht gänzlich überflüssig darauf hinzuweisen, dass die Begriffe ,Einheit' und ,Identität' in verschiedenen Kontexten der Kantischen Philosophie prominent sind, etwa wenn Kant von „Verstandeseinheit“ bzw. „Vernunfteinheit“ (KRV A 307 / B 363, A 645ff. / 673ff., u. ö.), Einheit der Anschauung (KRV A 79 / B 105, B 143, B 144 Anm., B 162, A 162 / B 203, u. ö.), Urteilseinheit (KRV A 69 / B 94), kategorial-quantitativer „Einheit“ (KRV A 80 / B 106) oder in seiner Auseinandersetzung mit Leibniz' principium identitatis indiscernibilium (z. B. KRV A 263f. / B 319f.) von numerischer Identität spricht. Solche und andere Bedeutungen werden in dieser Abhandlung nicht im einzelnen berücksichtigt, da es hier allein um Einheit und Identität des Bewusstseins gehen soll. Des weiteren sollte man sich über die Ambiguität der Kantischen Formal „Einheit des Bewußtseins“ im klaren sein. Zum einen wird „Einheit“ in diesem Ausdruck von Kant verstanden als Eigenschaft, die dem Bewusstsein selbst zukommt, in dem Sinne dass Bewusstsein ein ursprünglich einheitliches ist. Zum anderen versteht Kant unter „Einheit“ auch das vom Bewusstsein qua transzendentaler Apperzeption hervorgebrachte Produkt der Einheit als einer „Verbindung“ bzw. „Synthesis“ des „Mannigfaltigen“ (KRV B 129ff.). Diese Ambiguität besteht deshalb, weil Kant Bewusstsein und Selbstbewusstsein in transzendentaler Bedeutung als Spontanvermögen synthetisierender Handlungen des Verstandes begreift, das nicht nur selbst ursprüngliche Einheit ist, sondern diese auch unter gegebenen Vorstellungen herzustellen vermag. Die Einheit des Bewusstseins geht dabei überhaupt zurück auf die Diskursivität des Verstandes bzw. Begriffs.

\subsection{Die Diskursivitätsthese}

Gemäß der Diskursivitätsthese gründen Einheit und Identität des Bewusstseins in der Theorie des diskursiven Begriffs. Dabei geht es nicht um empirische Einheit und Identität wie sie einem Bewusstsein im Verlauf seiner mentalen 
Erlebnisgeschichte, etwa als Person, zukommen kann. Gegenstand der Diskursivitätsthese ist die Begründung der transzendentalen Einheit und Identität des Bewusstseins als Bedingung a priori der Erkenntnis. Die hier verteidigte Diskursivitätsthese ist also nicht zu verwechseln mit Allisons „discursivity thesis“, derzufolge menschliche Erkenntnis diskursiv ist, was nichts anderes heiße als ,it requires both concepts and sensible intuiton“ (Allison 1996: 6). Allisons „discursivity thesis" ist eine These über die Komposition von Erkenntnis. Kant setze sie letztlich als „ultimate ,fact'“ (ebd.) voraus, rechtfertige sie aber nicht eigens. Dies ist m. E. nicht der Fall. Was Kant als factum brutum nicht voraussetzt, sondern als kognitiven Befund über das menschliche Erkenntnisvermögen konstatiert, ist allein die Tatsache, dass der menschliche Verstand ein diskursives Vermögen ist. ${ }^{3}$ Dagegen behauptet die hier aufgestellte Diskursivitätsthese nichts in Bezug auf die Komposition von Erkenntnis, sondern führt die Struktur der Einheit des Bewusstseins auf die diskursive Struktur von Begriffen zurück. Während für Allison aus der „discursivity thesis“ der transzendentale Idealismus folgt, ${ }^{4}$ erklärt die Diskursivitätsthese also zunächst nichts über den Unterschied von Erscheinung und Ding an sich, auch wenn sich dieser $e x$ post daraus entwickeln lassen mag.

Mit der Diskursivitätsthese ist das Kantische Theorem kompatibel, dass Einheit eine ursprüngliche kognitive Eigenschaft des Bewusstseins ist. Denn die Diskursivitätsthese erklärt lediglich, warum Einheit eine kognitive Eigenschaft des Bewusstseins ist, nämlich weil sie auf diskursiven Begriffen beruht, und nicht warum sie eine ursprüngliche Eigenschaft des Bewusstseins ist. Das heißt Einheit kann eine ursprüngliche und insofern nicht reduzierbare kognitive Eigenschaft des Bewusstseins sein, wobei sich die Gründe ihres Bestehens durchaus angeben lassen.

Die Argumente für die Diskursivitätsthese lassen sich entwickeln im Ausgang vom Kantischen Grundtheorem, dass die Verbindung oder Synthesis eines wie auch immer gearteten Mannigfaltigen nicht einfach vorliegt, sondern durch das Bewusstsein bzw. den Verstand zustande gebracht werden muss und dass „Verbindung“ die „Vorstellung der synthetischen Einheit des Mannigfaltigen“ ist. Dabei entsteht die Vorstellung dieser Einheit nicht aus der Verbindung, sondern

${ }^{3}$ Zur Rechtfertigung des Kantischen Stämmedualismus vgl. Heidemann 2000. Siehe auch Düsing 2004: 104ff., der die Gründe erörtert, warum diskursive Begriffe ein sinnliches Anschauungsmannigfaltiges erfordern, um Erkenntnis möglich zu machen. ${ }^{4}$ Vgl. Allison 1996: 7. Zur „discursivity thesis“" siehe auch Allison 2004: 12ff., $77 \mathrm{ff}$. u. ö. 
macht diese erst möglich ( $K R V$ B 130 f.). Kant macht sein eigentliches Argument in einem Brief an Beck vom 1. Juli 1794 deutlicher als in der Kritik der reinen Vernunft:

„Die Zusammensetzung können wir nicht als gegeben warnehmen, sondern wir müssen sie selbst machen: wir müssen zusammensetzen, wenn wir uns etwas als zusammengesetzt vorstellen sollen (selbst den Raum und die Zeit). [...] Die Auffassung (apprehensio) des Manigfaltigen Gegebenen und die Aufnehmung in die Einheit des Bewustseyns desselben (apperceptio) ist nun mit der Vorstellung eines Zusammengesetzten (d.i. nur durch Zusammensetzung Möglichen) einerley, wenn die Synthesis meiner Vorstellung in der Auffassung, und die Analysis derselben so fern sie Begrif ist, eine und dieselbe Vorstellung geben [...].“(Briefe 11: 515).

Mit dieser Grundkonzeption, derzufolge „Erkenntnis“ in der Verstandeshandlung der „Synthesis“ besteht, nämlich darin, „verschiedene Vorstellungen zueinander hinzuzutun, und ihre Mannigfaltigkeit [...] zu begreifen" (KrV A 77 / B 103), legt sich Kant auf eine Epistemologie fest, die Erkenntnis als Produkt einer spezifischen Form reflektierender Tätigkeit und nicht als bloß kognitives Entdecken gegebener Sachverhalte begreift. ${ }^{5}$ Wenn Erkenntnis auf der Grundlage des Stämmedualismus von Sinnlichkeit und Verstand aber ein Reflexionsprodukt ist, muss gezeigt werden, wie der Verstand qua Apperzeption dieses Produkt aus einer Mannigfaltigkeit von Vorstellungen hervorbringt.

Da der Ort der Wahrheit gemäß Kant das Urteil ist und ein „Urteil nichts anderes sei, als die Art, gegebene Erkenntnisse zur objektiven Einheit der Apperzeption zu bringen“ (KRV B 142), spielen bei der Erzeugung der Einheit von Erkenntnis Begriffe eine maßgebliche Rolle: „Wir können aber alle Handlungen des Verstandes auf Urteile zurückführen, so daß der Verstand überhaupt als ein Vermögen zu urteilen vorgestellt werden kann. Denn er ist [...] ein Vermögen zu denken. Denken ist das Erkenntnis durch Begriffe.“ (KRV A 69 / B 94) ${ }^{6}$ Aus diesem

\footnotetext{
${ }^{5}$ Nach Allison (1996: 7) wäre letzteres gleichbedeutend mit der Suspension der „discursivity thesis“, da das kognitive Entdecken von Sachverhalten Formen a priori der Sinnlichkeit, die Gegebenes zuerst ordnen, überflüssig machen würde.

${ }^{6}$ Vgl. Heidemann 2004: $201 \mathrm{ff}$.
} 
Grunde gibt die Funktion und Struktur des Begriffs Aufschluss darüber, was ein Urteil ist und wie die objektive Einheit der Apperzeption zustande kommt.

Was also ist ein Begriff nach Kant? Der Kantischen Theorie zufolge ist ein Begriff anders als die Anschauung nicht einzelne (repraesentatio singularis), sondern allgemeine Vorstellung (repraesentatio universalis). Als solcher ist der Begriff zugleich „repraesentatio communis“ (Briefe 11: 348), das heißt eine Vorstellung, die nur aufgrund gemeinschaftlicher Merkmale gebildet werden kann. In der JäscheLogik heißt es: „Begriff [ist] eine allgemeine (repraesentatio per notas communes) oder reflectirte Vorstellung (repraesentatio discursiva).“ (Logik 9: 91). Neben der Allgemeinheit ist die Eigenschaft der Diskursivität von Begriffen entscheidend. Denn ein Begriff wird durch die Methode der Abstraktion gebildet und das heißt, dass Begriffsverhältnisse gemäß dem Modell der Subordination konzipiert werden müssen. Diskursiv sind Begriffe, weil sie dadurch gebildet werden, dass ein gegebenes Besonderes durchgegangen werden muss, um die ihnen gemeinsamen Merkmale unter Absehung vom sie Unterscheidenden herauszugreifen. Dabei breiten oder zerstreuen sich (von lat. discurrere) die gemeinsamen Merkmale als begriffliche Komplexionen im Besonderen. ${ }^{7}$ Begriffe sind insofern distributive Vorstellungen, die „einer unendlichen Menge von verschiedenen möglichen Vorstellungen (als ihr gemeinschaftliches Merkmal)“ zukommen und diese „unter sich“ enthalten (KRV A 25 / B 39f.). Begriffsverhältnissen liegt folglich eine nach Gattung und Art aufgebaute logische Ordnung zugrunde, in der es umfangreichere höhere und inhaltsärmere niedere Vorstellungen gibt. Ein Begriff ist also diskursiv allgemein, weil das Besondere oder Einzelne zunächst durchgegangen und daraufhin verglichen werden muss, was ihm gemeinsam ist: ,[...] denn durch das Allgemeine unseres (menschlichen) Verstandes ist das Besondere nicht bestimmt; und es ist zufällig, auf wie vielerlei Art unterschiedene Dinge, die doch in einem gemeinsamen Merkmale übereinkommen, unserer Wahrnehmung vorkommen können.“ (KU 5: 406). Das dabei abstrahierte, Vorstellungen gemeinschaftlich Zukommende, repräsentiert der Begriff als Komplexion von „Teilvorstellungen“ (KRV A 32 / B 48). So stellen

7 Auch wenn Abstraktion dabei eine methodische Rolle spielt, ist Kants Begriffstheorie doch keine reine Abstraktionstheorie, da Begriffen logische Funktionen zugrunde liegen (siehe unten). Dass Kants Begriffstheorie darüber hinaus gegenüber den klassischen Einwänden gegen die Abstraktionstheorie verteidigt werden kann, insbesondere gegen den Zirkeleinwand des Erwerbs von Begriffen, zeigt Vanzo 2012: 19-25, 133ff., 164ff. 
Begriffe das analytisch Identische dessen vor, was ansonsten verschieden ist. Kant versteht Begriffe demnach dezidiert nicht als Instrumente der Klassifikation bzw. Begriffsverhältnisse nicht als Klassifikationssysteme, die lediglich der Einteilung zum Beispiel von natürlichen Arten dienen. ${ }^{8}$

Da der menschliche Verstand nur mit Begriffen als allgemeinen Vorstellungen zu operieren vermag, verfährt er auch bei der Urteilsbildung auf diskursive Weise. Ein Begriff beruht nach Kant auf einer „Funktion“ als „Einheit der Handlung, verschiedene Vorstellungen unter einer gemeinschaftlichen zu ordnen.“ (KRV A 68 / B 93). Der Terminus „Funktion“ ist transzendentalphilosophisch zu verstehen als die Einheit stiftende, spontane Tätigkeit des Verstandes, Vorstellungen unter Begriffe zu bringen und dadurch Einheit unter ihnen herzustellen. Die durch Funktionen geregelte Verwendung von Begriffen bedeutet insofern nichts anderes als zu urteilen. Da durch einen Begriff als repraesentatio generalis ein Gegenstand nur mittelbar vorstellt wird, ist ein Urteil die „mittelbare Erkenntnis eines Gegenstandes, mithin die Vorstellung einer Vorstellung desselben.“ (KRV A 68 / B 93). Ein Urteil als „Vorstellung einer Vorstellung" kann darum nur durch ein diskursives Vermögen, den Verstand, erzeugt werden. Anders als die Sinnlichkeit stellt der menschliche Verstand nicht intuitiv, sondern mittels abstrakter Merkmale diskursiv vor. Aus diesem Grunde sind die Vorstellungen des Verstandes eben Allgemeines, das heißt sie repräsentieren analytische Identitäten von sonst vielfältig Verschiedenem und referieren daher nur mittelbar durch andere Vorstellungen auf Gegenstände. Kant erläutert dies in der Kritik der reinen Vernunft am Urteil „Alle Körper sind teilbar.“ (KRV A 68 / B 93). Der Begriff „Körper“ ist eine repraesentatio generalis, eine Vorstellung, die von vielem der gleichen Art gilt. Dies trifft auch auf den Begriff „Teilbarkeit“ (KRV A 69

\footnotetext{
${ }^{8}$ Dies ist bereits Kants Ansicht vor dem Erscheinen der Kritik der reinen Vernunft. In der kleinen Schrift Von den verschiedenen Racen der Menschen (1775) weist er ausdrücklich darauf hin, dass die Einteilung natürlicher Arten gemäß spezifischer Ähnlichkeitskriterien eine Klassifikation bloß für das „Gedächtniß“ (2: 429) darstellt, die - so kann vom Standpunkt der kritischen Lehre aus hinzufügen - nicht dem gerecht wird, was einen Begriff als diskursive Vorstellung auszeichnet. Aus diesem Grunde scheint mir Kitchers (1999: 368-369) Kennzeichnung der Kantischen Begriffstheorie als eine Theorie der Klassifikation („classification“) zumindest missverständlich, da darin die Eigenschaft der Diskursivität von Begriffen nicht zum Ausdruck kommt. Zur Einteilung nach Gattungen und Arten siehe auch Kants Lehre von „der Homogenität, der Spezifikation und der Kontinuität der Formen“ (KRV A 658 / B 686) im Abschnitt „Von dem regulativen Gebrauch der Ideen der reinen Vernunft" im Anhang zur transzendentalen Dialektik der Kritik der reinen Vernunft.
} 
/ B 94) zu, so dass dieses Prädikat ebenso von vielem der gleichen Art gilt. Kant meint nun, dass das Urteil „Alle Körper sind teilbar.“ nichts anderes ausdrückt, als dass durch den Begriff des „Teilbaren“ (KRV A 68 / B 93) bzw. der „Teilbarkeit“ neben anderem auch der Begriff „Körper“ vorgestellt wird und so bestimmte in der Anschauung gegebene „Erscheinungen“ (KRV A 69 / B 93), konkrete Körperdinge, durch das Prädikat der „Teilbarkeit“" mittelbar vorgestellt und erkannt werden können. In Urteilen werden also immer nur höhere Begriffe verwendet, die andere, umfangärmere Begriffe unter sich enthalten, um für „viele mögliche Erkenntnisse“ (KRV A 69 / B 94) gelten zu können.

Wenn nun Funktionen nichts anderes sind als Handlungen, „verschiedene Vorstellungen unter einer gemeinschaftlichen zu ordnen“, dann sind Urteile selbst nichts anderes als „Funktionen der Einheit unter unseren Vorstellungen“ (KRV A 68f. / B 93f.). In dem Urteil: „Alle Körper sind teilbar.“ ist somit die gesamte Sphäre des Subjektbegriffs unter den Prädikatbegriff subsumiert oder mittelbar durch den Prädikatbegriff vorgestellt, so dass durch diese Einheit gegebener Vorstellungen der Gedanke bzw. die Erkenntnis der Teilbarkeit aller Körper geformt werden kann. Der Begriff der Funktion lässt sich daher auch als Regel der Einheit der logischen Ordnung von Vorstellungen bezeichnen. Auch ein diskursiver Begriff muss auf einer logischen Ordnungsfunktion beruhen, da er indirekt-referenziell ist, das heißt sich auf die Gegenstände, die durch ihn vorgestellt werden, nur indirekt bezieht, indem Vorstellungen (Merkmale) zueinander in Subsumtionsbeziehungen gebracht werden. So wird durch die logische Funktion von Begriffen logische Ordnung unter Vorstellungen möglich, wodurch Begriffe selbst ihre Einheit erhalten.

Einheit ist insofern zunächst eine logische Eigenschaft von Begriffen. Eine logische Eigenschaft ist eine Eigenschaft, die der Form und nicht dem Inhalt eines Begriffs zukommt. Gleich welchen Inhalt ein Begriff hat, logisch-formal stellt er immer eine Einheit dar. Dabei geht es nicht darum, wie Begriffe gebildet werden, etwa durch Abstraktion, sondern um die grundlegendere Frage der formalen Erzeugung eines Begriffs. Denn um einen Begriff durch Abstraktion von Merkmalen bilden zu können, muss man schon wissen, wie ein Begriff überhaupt formal, also diskursiv erzeugt wird. Ein Begriff ist dabei selbst eine logisch-diskursive Einheit. ${ }^{9}$

\footnotetext{
${ }^{9}$ In der Jäsche-Logik erläutert Kant dies eher schematisch anhand der „logischen Verstandes-Actus" der „Comparation, Reflexion und Abstraction“: „1) die Comparation, d.i. die Vergleichung der Vorstellungen unter einander im Verhältnisse
} 


\subsection{Diskursivität und Einheit des Bewusstseins}

Die erforderliche Begründung für den kognitiven Sachverhalt, dass ein Begriff formal eine logisch-diskursive Einheit darstellt, liefert der der Kantischen Theorie des Selbstbewusstseins entstammende „Grundsatz“ (KRV B 136) der ursprünglichen „Einheit des Bewußtseins“. Die ursprüngliche Einheit des Bewusstseins ist Bedingung der Möglichkeit dafür, einen Begriff haben zu können. Im einzelnen gezeigt wird dies im erstes Beweisschritt der transzendentalen Deduktion der reinen Verstandesbegriffe. Das Argument beginnt mit dem bereits angeführten Theorem, dass „wir uns nichts, als im Objekt verbunden, vorstellen können, ohne es vorher selbst verbunden zu haben“, da die „Verbindung“ eines Mannigfaltigen durch die spontane „Verstandeshandlung“ der „Synthesis“ erst erzeugt wird. Die Möglichkeit der Verbindung aber setzt die apriorische „Vorstellung der synthetischen Einheit des Mannigfaltigen“ voraus (KRV B 130f.). „Grund“ dieser „Einheit“, die nach Kants ausdrücklichem Hinweis nicht mit der „Kategorie der Einheit“ (KRV B 131) verwechselt werden darf, ist die ,ursprünglich-synthetische[...] Einheit der Apperzeption“. Als „transzendentale Einheit des Selbstbewußtseins“ (KRV B 131) sei sie Möglichkeitsbedingung des Bewusstseins mannigfaltiger Vorstellungen, und zwar indem sie es als ,analytische Einheit der Apperzeption“ möglich macht, dass ein und dasselbe „Ich denke“-Bewusstsein mannigfaltige Vorstellungen sowohl haben als auch durch „Synthesis“ zur Einheit verbinden kann. Dadurch stellt die Apperzeption die „Identität des Bewußtseins in diesen Vorstellungen selbst“" vor (KRV B 133). Als Folgerung aus dem Verbindungs-Theorem setzt dieses analytische Identitätsbewusstsein aber seinerseits die Möglichkeit synthetischer Einheit von Vorstellungen voraus, nämlich durch die ,ursprüngliche synthetische Einheit der Apperzeption“ ( $K R V$ B 135). So beruht letztlich alle Einheit von Vorstellungen nach Kant auf der „synthetische[n] Einheit des Bewußtseins“, die zugleich, „objektive Einheit des Selbstbewußtseins“ ist (KRV B 137ff.). Diese Einheit des Selbstbewusstseins ist rein logisch, das heißt sie wird nicht psychisch in der Zeit konstituiert und beruht nicht auf einem kontingenten Faktum der Synthesis von Vorstellungen. Sie ist transzendentale Einheit.

zur Einheit des Bewußtseins; 2) die Reflexion, d.i. die Überlegung, wie verschiedene Vorstellungen in Einem Bewußtsein begriffen sein können; und endlich 3) Die Abstraction oder die Absonderung alles Übrigen, worin die gegebenen Vorstellungen sich unterscheiden.“(Logik 9: 94). 
Wenn also die Einheit von Vorstellungen auf die synthetische Einheit der Apperzeption zurückgeht, beruht auch die mittels der logischen Funktion erzeugte Einheit des Begriffs auf der Einheit des Selbstbewusstseins. Dass sich dies so verhält, zeigt die notorisch schwierige Anmerkung zur analytischen Einheit des Selbstbewusstseins der transzendentalen Deduktion:

„Die analytische Einheit des Bewußtseins hängt allen gemeinsamen [allgemeinen; D.H.] Begriffen, als solchen, an, z.B. wenn ich mir rot überhaupt denke, so stelle ich mir dadurch eine Beschaffenheit vor, die (als Merkmal) irgend woran angetroffen, oder mit anderen Vorstellungen verbunden sein kann; also nur vermöge einer vorausgedachten möglichen synthetischen Einheit kann ich mir die analytische vorstellen. Eine Vorstellung, die als verschiedenen gemein gedacht werden soll [ein Begriff; D.H.], wird als zu solchen gehörig angesehen, die außer ihr noch etwas Verschiedenes an sich haben [z.B. der Begriff ,Baum': Erlen, Buchen, Linden; D.H.], folglich muß sie in synthetischer Einheit mit anderen (wenn gleich nur möglichen Vorstellungen) vorher gedacht werden, ehe ich die analytische Einheit des Bewußtseins, welche sie zum conceptus communis macht, an ihr denken kann.“ (KRV B 133f. Anm.)

Wenn nach Kant die analytische Einheit der Apperzeption den Begriff möglich macht, ist dies so zu verstehen, dass um einen Begriff als diskursive Vorstellung, das heißt als Vorstellung eines Merkmals bzw. einer Komplexion von Merkmalen konzipieren zu können, auch der Gedanke gefasst werden können muss, dass ein Merkmal anderen Vorstellungen als das in ihnen analytisch Identische zukommt. Wie gesehen, ist dieser Gedanke nur möglich durch die analytische Einheit der Apperzeption, die ihrerseits nur möglich ist aufgrund der synthetischen. Um zum Beispiel den Begriff „Blume“ haben zu können, muss ich wissen, was es bedeutet, dass die durch diesen Begriff vorgestellten Merkmale in den mannigfaltigen einzelnen Blumen (Lilien, Tulpen, Rosen usw.) dieselben sind. Diese Vorstellung ist die einer analytischen Identität von ansonsten Verschiedenem, die durch das Selbstbewusstsein nur als analytische Einheit begriffen werden kann. Diese vorgestellte Einheit, der Begriff, impliziert dabei Synthesis, nämlich die Verbindung der von anderen Vorstellungen abstrahierten Merkmale zum Begriff. Der Akteur dieser Synthesisleistungen, durch die die analytische Einheit zustande gebracht wird, ist die 
Apperzeption, und die Apperzeption ist der „Verstand selbst“ (KRV B 134 Anm.) als der kognitive Ursprung von Einheit. Wer also weiß, was es heißt, einen Begriff zu haben bzw. wer ein kompetenter Begriffsverwender ist, weiß implizit auch, dass Begriffe diskursiv-allgemeine Vorstellungen und als solche analytisch-identische Einheiten sind. Und wenn eine Person dies weiß, besitzt sie ebenfalls das Wissen, dass es höhere und niedere Begriffe als Gattungen und Arten gibt, jede Art selbst wieder Begriff ist, der als Gattung fungieren kann usw., da sie andernfalls einen Begriff gar nicht formal erzeugen könnte. Die Fähigkeit, Begriffe zu haben, sie also $\mathrm{zu}$ erzeugen und $\mathrm{zu}$ verwenden, ist somit eine an das Selbstbewusstsein gebundene kognitive Fähigkeit, deren Ausübung ein Wissen über die logisch-formale Struktur sowie das diskursiv-analytische Verhältnis von Begriffen zueinander impliziert.

Analog zum Begriff gilt dabei für das Urteil, zum einen dass es eine logische Ordnung von Vorstellungen enthält, die eine durch Funktionen geregelte Einheit darstellt, sowie zum anderen dass es allgemeinere und besondere Urteile gibt wie ,Alle Lilien sind Blumen.' und ,Einige Blumen sind Lilien.' Die Fähigkeit, solche Urteile bilden zu können, impliziert folglich wie im Falle des Begriffs ein Wissen um die logisch-hierarchische Über- und Unterordnung von Urteilen. Das heißt wenn ich das Urteil bilden kann ,Alle Lilien sind Blumen.', dann muss ich auch das diesem gegenüber besondere Urteil bilden können ,Einige Blumen sind Lilien.' und darüber hinaus annehmen, dass sich die Gattung ,Blume' in weitere Arten einteilen lässt, die wiederum Unterarten haben, usw.

Die durch die synthetische Einheit der Apperzeption geforderte logische Identität des Selbstbewusstseins ist also deswegen eine analytische, weil sie sich als diskursive Identität erweist. Und diskursive Identität ist sie, weil die Apperzeption nichts anderes ist als der „Verstand selbst“, nämlich das Vermögen der Begriffe, in denen, wie Kant auch formuliert, „eben dasselbe Bewusstsein, als in vielen Vorstellungen“" enthalten gedacht wird (KRV B 137 Anm.). Das heißt also, dass Kants transzendentale Theorie des Selbstbewusstseins, genauer der Zusammenhang zwischen synthetischer und analytischer Einheit der Apperzeption in der kritischen Theorie des diskursiven Begriffs gründet. Denn die Identität der Apperzeption ist deswegen eine analytische, weil sie sich als eine diskursive erweist. Hierbei handelt es sich um einen kontingenten kognitiven Sachverhalt, da die Identität der Apperzeption ja ebenso als eine synthetische konzipiert werden könnte, so dass sich Identität, also „dasselbe Bewusstsein, als in vielen Vorstellungen“, nicht aus der 
Reflexion des Verstandes, sondern durch Anschauung, also durch „viel(e) Vorstellungen als in einer, und deren Bewusstsein, enthalten" (KRV B 137, Anm.) ergäbe. Das menschliche Erkenntnisvermögen jedoch ist kein solches, dem das Prinzip der synthetischen Identität des Selbstbewusstseins, sondern dasjenige der diskursiv-analytischen Identität eigentümlich ist. ${ }^{10}$ Dass die Theorie des transzendentalen, reinen Selbstbewusstseins in der Begriffstheorie gründet, heißt also: Wären diskursive, allgemeine Vorstellungen, das heißt Begriffe, für den menschlichen Verstand keine Bedingungen des Denkens und Erkennens, könnte der menschlichen Apperzeption weder synthetische Einheit noch analytische Identität zukommen, zumindest nicht auf eine für die menschliche Rationalität nachvollziehbare Weise. Denn da der menschliche Verstand nicht anschaut, sondern denkt, und sich Denken für den menschlichen Verstand nicht anders vollziehen kann als durch den Gebrauch von Begriffen, die wiederum auf diskursiver Einheit beruhen, hängen Einheit und Identität des Bewusstseins von der Diskursivität des Begriffs als unhintergehbarer formaler Struktur des Denkens ab. Eine Erkenntnis personaler Einheit und Identität wird damit jedoch nicht erreicht, wie nun zu zeigen ist.

\section{Einheit und Identität der Person}

Das soeben explizierte Verhältnis von logischer Einheit und diskursiver Identität des Bewusstseins ist kein begriffliches Glasperlenspiel, sondern hat als transzendentale Bedingung der Möglichkeit von Erkenntnis objektive Bedeutung. Der konstitutive Zusammenhang von Einheit und Identität des Bewusstseins ist in einem weiteren Kontext relevant, im dritten Paralogismus der Personalität, so dass man annehmen könnte, auch in diesem Fall würden Einheit und Identität des Bewusstseins objektive Erkenntnisbedeutung besitzen, nämlich dass der Seele aufgrund der Einheit und diachronen Identität ihres Bewusstseins der Status des Personseins zukommt. Dies ist nach Kant jedoch gerade nicht der Fall. Um die Diskursivitätsthese und den mit ihr verbundenen Begriff der diskursiven Identität weiter zu erhellen, wird im Folgenden auf die Unterschiede zwischen diskursiver und personaler Identität

\footnotetext{
${ }^{10}$ Vgl. KRV B 135: „Dieser Grundsatz, der notwendigen Einheit der Apperzeption, ist nun zwar selbst identisch, mithin ein analytischer Satz, erklärt aber doch eine Synthesis des in einer Anschauung gegebenen Mannigfaltigen als notwendig, ohne welche jene durchgängige Identität des Selbstbewußtseins nicht gedacht werden kann.“
} 
eingegangen und es werden die Gründe eruiert, warum aus der Einheit des Bewusstseins nichts in Bezug auf die Eigenschaft des Personseins folgt.

Der dritte Paralogismus der ersten Auflage der Kritik der reinen Vernunft (A 361) hat folgenden Wortlaut:

„(1) Was sich der numerischen Identität seiner selbst in verschiedenen Zeiten bewußt ist, ist so fern eine Person:

(2) Nun ist die Seele [sich der numerischen Identität ihrer selbst in verschiedenen Zeiten bewußt ist; D.H.].

Also ist sie eine Person.“

In seiner Kritik des dritten Fehlschlusses der rationalen Seelenlehre (KRV A 361-366) hebt Kant darauf ab, dass der Obersatz dieses Schlusses eigentlich nichts anderes als ein ,identischer [analytischer; D.H.] Satz des Selbstbewusstseins in der Zeit" (KRV A 362) ist. Denn zu jedem Zeitpunkt bin ich das identische Bewusstsein meiner Vorstellungen. Die zu klärende Frage lautet nun, ob ich auch zu verschiedenen Zeitpunkten das identische Bewusstsein meiner Vorstellungen bin und wie sich dies erkennen ließe. Sollte sich die diachrone Identität meines Bewusstseins, wie klassische metaphysica specialis behaupten muss, aus begrifflichen Gründen objektiv erkennen lassen, so würde sich aus dem rein begrifflichen Zusammenhang von Einheit und Identität des Bewusstseins ein wahrer Sachverhalt ableiten lassen, nämlich dass die Seele als Person existiert. Diese Schlussfolgerung aber geht weit über das hinaus, was sich anhand der Analyse der Diskursivitätsthese ergab und objektiv begründbar ist.

\subsection{Zwei Gedankenexperimente: Der äußere und innere Beobachter}

Um die Frage zu beantworten, ob ich auch zu verschiedenen Zeitpunkten das identische Bewusstsein meiner Vorstellungen bin und vor allem wie dies aus reinen Begriffen erkannt werden könnte, unterscheidet Kant in seiner Kritik des dritten Paralogismus zwei generelle Antwortoptionen, die er jeweils in Form eines Gedankenexperiments darstellt. Im ersten, dem Gedankenexperiment des äußeren Beobachters, wird überlegt, ob ein ,äußere[r] Beobachter“ (KRV A 362) die Identität meines Bewusstseins, verstanden als die zeitliche Beharrlichkeit meiner Seele, sozusagen drittpersonal von außen feststellen könnte, zum Beispiel indem er meine 
Bewusstseinszustände extern erkundet und bezeugt, es handle sich offenkundig um die wechselnden mentalen Zustände desselben Bewusstseinsinhabers. Dazu ist er nach Kant nicht in der Lage. Denn der Zeitraum, in den er mein Bewusstsein als identisch setzt, wäre ja gar nicht der Zeitraum meines Bewusstseins, sondern der seines eigenen beobachtenden Bewusstseins. Ein äußerer Beobachter würde nämlich stets nur konstatieren können, zu einem bestimmten Zeitpunkt $t_{l}$ eine Beobachtung zu machen, also eine Vorstellung zu haben, deren Gegenstand mein Bewusstsein in der Zeit ist. Zum Zeitpunkt $t_{2}$ kann er zwar eine weitere Vorstellung haben, deren Gegenstand mein Bewusstsein in der Zeit ist, aber aus diesem Sachverhalt kann er nur schließen, dass er zum Zeitpunkt $t_{1}$ sowie zum Zeitpunkt $t_{2}$ jeweils mein Bewusstsein in der Zeit zum Gegenstand seiner Vorstellung gemacht hat. Daraus ergibt sich nicht die numerische Identität des vorgestellten Bewusstseins und ebenso wenig, wie man ergänzen kann, die Identität des vorstellenden Bewusstseins des äußeren Beobachters:

„Denn [...] in der ganzen Zeit, darin ich mir meiner bewußt bin, bin ich mir dieser Zeit, als zur Einheit meines Selbst gehörig, bewußt, und es ist einerlei, ob ich sage: diese ganze Zeit ist in Mir als individueller Einheit, oder ich bin mit numerischer Identität, in aller dieser Zeit befindlich.“ (KRV A 362)

Aus der externen Perspektive der dritten Person kann personale Identität also nie festgestellt werden, da der äußere Beobachter immer nur von seiner eigenen auf meine personale Identität schließen würde. Denn ein äußerer Beobachter könnte keine Informationen über einen erstpersonalen zeitlichen Vorstellungswechsel in mir haben und folglich nichts über die numerische Identität meines Bewusstseins wissen. ${ }^{11}$

Wenn überhaupt, kann personale Identität nur aus der Perspektive der ersten Person erkannt werden, wie Kant in einem zweiten, dem Gedankenexperiment des inneren Beobachters ausführt: Nun wird argumentiert, dass die Identität meines Selbstbewusstseins in verschiedenen Zeiten lediglich die formale Bedingung der Einheit meines Bewusstseins ist, aber nichts über die numerische Identität meines Subjekts aussagt. Kant veranschaulicht sein Argument wie folgt: Nehmen wir eine sukzessive Reihe von (geistigen) Substanzen an, wobei die erste der zweiten, die zweite der dritten usw. jeweils ihr gesamtes Bewusstsein übertrüge. Die letzte

${ }^{11}$ Kant geht hier davon aus, dass die eigenen mentalen Zustände nur erstpersonal zugänglich sind. 
Substanz dieser Reihe würde sich dann aller mentalen Zustände der Vorgängersubstanzen als ihrer eigenen bewusst sein. Aber dennoch, so Kant, diese letzte Substanz würde doch nicht die identische, das heißt dieselbe Person in allen diesen Zuständen gewesen sein, selbst wenn sie sich aller ihr übertragenen Gedankeninhalte bewusst sein sollte (KRV A 363 f, Anm.).

Aus der Tatsache also, dass ich eine kontinuierliche Erinnerung an meine mentale Vergangenheit habe, folgt nicht, dass ich in diesem Erinnerungszeitraum dieselbe Person bin bzw. war. Gemäß Kant ist zumindest denkbar, dass ich mir in meiner Erinnerung nur der Summe von Gedankeninhalten anderer Subjekte bewusst bin. Damit die Erkenntnis meiner selbst als eines zeitlich beharrenden Selbst aus erstpersonaler Perspektive möglich wäre, müsste ich mich in meinem Bewusstsein mit mir selbst vergleichen können, und zwar so, dass ich mich selbst als den in der Zeit beharrenden Bezugspol meiner wechselnden Vorstellungen beobachten könnte. Eben dies ist nach Kant nicht möglich, denn was das Bewusstsein dabei beobachten würde, wäre lediglich es selbst als das analytisch identische Bewusstsein seiner Vorstellungen. Um die Identität meines Bewusstseins feststellen zu können, müsste ich mich in meinem Bewusstsein aber als etwas objektiv Beharrliches in der Zeit erkennen können. Eine solche, wie Kant sie nennt, „Selbsterkenntnis durch reine Vernunft [...] aus dem bloßen Begriffe des identischen Selbst“" (KRV A 366) ist nicht möglich, da wir nicht, wenn man so will, aus uns heraustreten können, um unseren Vorstellungswechsel gleichsam von außen $\mathrm{zu}$ betrachten. Wie das Gedankenexperiment des äußeren Beobachters bereits gezeigt hat, lässt sich personale Identität darüberhinaus generell nicht aus einer externen Perspektive erkennen. ${ }^{12}$

Mit dem Gedankenexperiment des inneren Beobachters bezieht sich Kant möglicherweise konkret auf Lockes Konstitutionstheorie personaler Identität und den darin zum Tragen kommenden Zusammenhang zwischen Einheit und Identität des Bewusstseins. Auch für Locke ist ,Einheit' eine wesentliche Eigenschaft des Bewusstseins. ${ }^{13}$ In seiner Theorie unterscheidet Locke zwischen Mensch, Substanz

\footnotetext{
${ }^{12}$ In der Anthropologie nennt Kant das „Beobachten seiner selbst“ „,eine methodische Zusammenstellung der an uns selbst gemachten Wahrnehmungen, welche den Stoff zum Tagebuch eines Beobachters seiner selbst abgiebt und leichtlich zu Schwärmerei und Wahnsinn hinführt.“ (Anth 7:132). Zu theoretischer Erkenntnis reicht die Selbstbeobachtungmethode also nicht hin. Vgl. Fortschritte 20: 270.

${ }^{13}$ Dem Begriff der Einheit kommt im Kapitel „Of Identity and Diversity“ (II.27) des An Essay Concerning Human Understanding nicht nur im Kontext der Erörterung der
} 
und Person (Essay II 27, 7). Der Begriff ,Mensch’ („man“, Essay II 27, 6 u. 8) referiert dabei auf den Körper eines bestimmten Menschen. Identitätskriterium des menschlichen Körpers sei die Organisation seiner Glieder zur Teilnahme an einem gemeinsamen Leben. Der Begriff ,Substanz’ (,substance“, Essay II 27, 2) bezeichnet generell den Träger von Eigenschaften. Da dieser Begriff undeutlich ist, können wir jedoch nicht wissen, was die Identität einer Substanz ausmacht. Demgegenüber bedeutet „person“ „a thinking intelligent being, that has reason and reflection, and can consider itself as itself“" (Essay II 27, 9). Als denkendes, intelligentes Wesen zeichnet sich die Person durch Bewusstsein bzw. Selbstbewusstsein aus. Mithilfe der Begriffe des Selbstbewusstseins und Bewusstseins lässt sich allererst erklären, und darauf kommt es an, wie ein denkendes Wesen wissen kann, an verschiedenen Orten und zu verschiedenen Zeiten als dasselbe zu denken. Denn durch die reflexive Struktur des Bewusstseins als Selbstbewusstsein wird das ,Ich' und damit die Identität der Person konstituiert, und zwar indem das Bewusstsein durch Reflexion, das heißt mittels Selbstbezug, oder Selbstbewusstsein, und anhand des Erinnerungskriteriums feststellt, zu einem früheren Zeitpunkt dasselbe Bewusstsein wie zu einem späteren bzw. zum gegenwärtigen Zeitpunkt $\mathrm{zu}$ sein. Personale Identität muss daher nach Locke verstanden werden als das von einem Bewusstsein aufgrund von Erinnerungsleistungen konstituierte Selbst, das sich in der Erinnerung aufgrund der Selbstzuschreibung seiner früheren Handlungen der zeitlichen Kontinuität seiner Existenz bewusst ist. Dabei ist die denkende Substanz zwar unverzichtbare Realisationsgrundlage personaler Identität, doch spreche nichts dagegen, dass sich die Identität ein und derselben Person in unterschiedlichen Substanzen realisiert. Was gemeint ist, geht aus dem Beispiel hervor, das Locke gibt:

Personbegriffs eine grundlegende Funktion zu. Auch bei der Festlegung des Identitätskriteriums zum Beispiel von Pflanzen, operiert Locke mit dem Begriff der Einheit, so dass etwa die Einheit der Teile einer Eiche diese zu einer Pflanze macht (vgl. Essay II.27, 4). Ebenso gilt für den menschlichen Körper („man“), dass dieser deswegen ein solcher ist, weil seine Teile in ihrem Zusammenhang eine Einheit bilden. Grundlegend ist ,Einheit' für das Selbstbewusstsein, das nicht ursprünglich ist, sondern durch die Erinnerung die Einheit mentaler Zustände herstellt (,uniting“, Essay II.27, 10). Denn „Consciousness alone unites actions into the same person.“ (Essay II.27, 16; vgl. 23-25). Lockes Essay wird unter Angabe des Buches, Kapitels und Paragraphen in modernisierter Schreibweise zitiert nach der Ausgabe von P. H. Nidditch (Hrsg.), Oxford 1975. 
,$[\ldots]$ it must be allowed, that, if the same consciousness [...] can be transferred from one thinking substance to another, it will be possible that two thinking substances may make but one person. For the same consciousness being preserved, whether in the same or different substances, the personal identity is preserved.“ (Essay II 27, 13). ${ }^{14}$

Der für Locke entscheidende Punkt ist, dass es keine Rolle spielt, in welcher Substanz ein Bewusstsein denkt. Es komme allein darauf an, dass ein Bewusstsein als Selbstbewusstsein mithilfe des Erinnerungskriteriums die Identität einer Person konstituiere. An einen solchen oder ähnlichen Fall denkt Kant im Gedankenexperiment des inneren Beobachters, das den Transfer von Bewusstseinsinhalten von Substanz zu Substanz annimmt. Den auch von Locke angenommenen Zusammenhang von Einheit und Identität des Bewusstseins stellt Kant nicht grundsätzlich in Frage: „Daß der Mensch in seiner Vorstellung das Ich haben kann, erhebt ihn unendlich über alle andere auf Erden lebende Wesen. Dadurch ist er eine Person und vermöge der Einheit des Bewußtseins bei allen Veränderungen, die ihm zustoßen mögen, eine und dieselbe Person“ (Anth 7:127) Insofern die Einheit des Bewusstseins Ermöglichungsbedingung personaler Identität ist, würde Kant dem Lockeschen Vorschlag sogar ausdrücklich zustimmen. Allerdings handelt es sich in diesem Falle nicht um diskursiv-logische, sondern um diachrone Identität, die sich als materiale Bestimmung nicht aus der Formalbestimmung der Einheit des Bewusstseins ableiten lässt. Denn auch wenn mir der Einheitszusammenhang im Bewusstsein einer reichhaltig bestimmten mentalen Geschichte, die ich mir selbst zuschreibe, formal

\footnotetext{
${ }^{14}$ Brook 1994: 38 scheint eine solche theoretische Möglichkeit auch Kant zuschreiben zu wollen, wenn er „Einheit des Bewusstseins“ definiert als: „(i) a single act of consciousness, which (ii) makes one aware of a number of representations and/or objects of representation in such a way that to be aware of any of this group is also to be aware of at least some others in the group and as a group." Denn aus dieser Definition schließt er, ,it is possible to have one, unified system of awareness without being one mind and certainly without being one person [...]. At least for identity across time, Kant also argued for this possibility [...]. Unity of consciousness may require identity of consciousness (B134), and identity of mind may require unity of consciousness (A108), but Kant nowhere says that unity of consciousness requires identity of mind." Brook zufolge ist es nach Kant offenbar denkbar, dass sich wie bei Locke die Einheit des Bewusstseins in unterschiedlichen Trägersubstanzen realisieren kann. Wie im dritten Paralogismus würde Kant eine solche Erwägung wohl als metaphysische Spekulation zurückweisen.
} 
vorliegt, folgt daraus nicht, dass ich in diesem Zusammenhang das durch Erinnerung konstituierte identische Erlebnissubjekt dieser Geschichte bin, wie Locke annimmt. ${ }^{15}$

\subsection{Numerische Identität und personale Einheit des Bewusstseins}

Während also, wie gesehen, diskursive Identität des Bewusstseins objektive Erkenntnisbedeutung hat, ist numerisch-diachrone, verstanden als personale Identität, untauglich, um aus der Selbigkeit des Bewusstseins zu jedem aktualen Zeitpunkt des Vorstellens auf die Selbigkeit des Bewusstseins zu verschiedenen Zeitpunkten des Vorstellens zu schließen. Letzteres ist, so Kants Argument, ein ,identischer Satz des Selbstbewusstseins in der Zeit" (KRV A 362), und zwar insofern aus der ersten Prämisse des dritten Paralogismus, also aus numerischer qua personaler Identität des Bewusstseins, eben nichts anderes folgt als die Tautologie, dass ich zu jedem Zeitpunkt meines Vorstellens derselbe Vorstellende bin. Zwar setzt auch numerische qua personale Identität Einheit des Bewusstseins voraus, zum Beispiel im Gedanken des ,Ich bin Ich', doch bleibt diese jeweils punktuell und bedeutet keine notwendige synthetische Einheit der Identität des Bewusstseins zu verschiedenen Zeiten. Der für den Begriff der Person relevante Identitätsbegriff ist mithin nicht derjenige der diskursiven Identität, die sich als logische Eigenschaft von Begriffen erwies. Der Gedanke, ich bin zu jedem Zeitpunkt, an dem ich die Vorstellung $x$ habe, das identische Subjekt dieser Vorstellung' ist zwar eine distributive Vorstellung, von der analytisch wahr ist, dass sie auf jede mentale Instantiierung meines Habens einer Vorstellung zutrifft. Denn hierbei handelt es sich um ein analytisches Subordinationsverhältnis von Vorstellungen, wie es für jeden diskursiven Begriff gilt. Die daraus abgeleitete Schlussfolgerung, ich sei auch zu verschiedenen Zeitpunkt, an denen ich die Vorstellung $x$ habe, das identische Subjekt dieser Vorstellungen', ist jedoch unzulässig, weil sie einen materialen Zusammenhang unter den Instantiierungen meines Bewusstseins von Vorstellungen herstellt, der in dem analytisch wahren Satz über die Identität des Bewusstseins zu jedem Zeitpunkt des Vorstellens nicht enthalten ist. Aus diesem Grunde hat der als solcher nicht zu bestreitende Zusammenhang von Einheit des Bewusstseins und diachroner Identität der Person keine objektive Erkenntnisbedeutung.

\footnotetext{
${ }^{15}$ Siehe zu den relevanten Unterschieden zwischen Locke und Kant Baum 2002: 109f., 119f. Vgl. auch Thiel 2015: 159ff.
} 
Wie die beiden Gedankenexperimente der Konstruktion personaler Identität aus dritt- und erstpersonaler Perspektive zeigen, wird im dritten Paralogismus zu unrecht auf die synthetische Einheit der Person geschlossen, weil der Zusammenhang zwischen Einheit und Identität hier kein logischer ist, sondern auf der zeitlichmentalen Kontinuität des Bewusstseins beruht. Dagegen ist die synthetische Einheit der Apperzeption eine notwendige, ahistorische, weil die analytische Einheit der Apperzeption auf logisch-diskursiver Identität beruht. Obwohl zwischen Einheit und Identität des Bewusstseins in der transzendentalen Deduktion sowie im dritten Paralogismus der prima facie gleiche begriffliche Konstitutionszusammenhang besteht, erweist sich dieser nur im ersteren Fall als legitimierbar. Hinsichtlich Einheit und Identität des Bewusstseins muss daher unterschieden werden zwischen atemporaler, notwendiger logischer Einheit und diskursiver Identität im Rahmen des transzendentalphilosophischen Projekts einerseits, und temporaler, kontingenter Einheit und personaler Identität im Rahmen des rationalistischen Projekts einer Metaphysik der Person andererseits.

\section{Fazit}

In diesem Beitrag wurden Argumente für die Diskursivitätsthese entwickelt. Die Diskursivitätsthese besagt, dass die spezifische Art der Einheit und damit zugleich die Identität menschlichen Bewusstseins in der Diskursivität des Begriffs bzw. des Verstandes gründet. Alleinige kognitive Operationsgrundlage des menschlichen Verstandes sind Begriffe als allgemeine, diskursive Vorstellungen, durch deren Gebrauch in Urteilen transzendentale Einheit und Identität des Bewusstseins zustandekommen. Anders als durch die Synthesis diskursiver Vorstellungen lässt sich nicht erklären, wie Einheit und Identität des Bewusstseins konstituiert werden. Die ursprüngliche Einheit des Bewusstseins stellt sich also, so könnte man schlussfolgern, als ursprüngliche Diskursivität des Verstandes heraus.

Damit tritt allerdings ein grundsätzliches Bedenken gegenüber der Diskursivitätsthese zutage, das bereits kurz erwähnt wurde. Wenn die Diskursivität des Verstandes als kognitive Tatsache die Einheit des Bewusstseins begründet, wie kann die Einheit des Bewusstseins dann ,ursprünglich“ sein (KRV A 118; B 131, 136, 151)? Denn was ursprünglich ist, kann nicht auf Grundlegenderes zugeführt und durch es begründet werden. Ist es nicht vielmehr so, dass die Diskursivität des Begriffs bzw. des Verstandes in der Einheit des Bewusstseins gründet? Dieses 
Bedenken ist ernst zu nehmen, lässt sich aber mit dem Hinweis darauf zurückweisen, dass die Diskursivitätsthese nicht beansprucht, eine Grundlegung der Ursprünglichkeit der Einheit des Bewusstseins zu liefern, sondern deren diskursive Struktur zu erklären. Als solche ist Diskursivität zwar fundamental für die Einheit des Bewusstseins, da ihre Konstitution und Struktur ohne Diskursivität nicht einsehbar wäre. Dass aber „die synthetische Einheit der Apperzeption der höchste Punkt [ist], an dem man allen Verstandesgebrauch, selbst die ganze Logik und nach ihr die Transzendental-Philosophie heften muß“ (KRV B 134 Anm.), wird dadurch nicht in Frage gestellt. Das heißt die Einheit des Bewusstseins bleibt eine ursprüngliche, auch wenn die Diskursivitätsthese zeigt, warum sie Einheit einer ganz bestimmten, eben diskursiven Art ist.

$\mathrm{Zu}$ einer so weitreichenden Schlussfolgerung, wie Brook sie zieht, gibt die Diskursivitätsthese allerding keinen Anlass: „Kant concluded by arguing that for unified awareness to be possible, objects or contents of conceptualized representation must be synthesized under concepts.“ (Brook 1994: 39). Es ist nicht ganz klar, ob Brook damit einen starken Konzeptualismus in Kauf nimmt, demzufolge alles einheitliche Bewusstsein immer schon unter Begriffen steht. Die Diskursivitätsthese impliziert keine solche Behauptung, sondern restringiert die diskursive Struktur des Bewusstseins auf kognitive Zustände, in denen die Einheit des Bewusstseins transzendentale Bedingung der Erkenntnis ist. Jenseits der transzendentalen Einheit bleibt die Möglichkeit nicht-begrifflichen Inhalts erhalten. Die Diskursivitätsthese ist folglich vereinbar mit Kants Non-Konzeptualismus. ${ }^{16}$

Auf der anderen Seite scheint die Diskursivitätsthese Ähnlichkeit mit dem zu haben, was insbesondere seit Patrica Kitchers Kant's Transcendental Psychology (1990) als funktionalistische Lesart der Kantischen Erkenntnistheorie diskutiert und von Andrew Brook in Kant and the Mind weitergeführt wurde. Gemäß der funktionalistischen Lesart werden die kognitiven Funktionen aus der Analyse und Beobachtung von kognitiven Aktivitäten des menschlichen Erkenntnisvermögens erschlossen und nicht unmittelbar durch das Bewusstsein erfasst. Dabei erkennt sich der menschliche Geist nicht wie er an sich ist, sondern - hier ganz in Übereinstimmung mit Kant - nur wie er sich in der Ausübung seiner kognitiven

\footnotetext{
${ }^{16} \mathrm{Zu}$ Argumenten für Kants Non-Konzeptualismus siehe Heidemann 2013.
} 
Funktionen selbst erscheint. ${ }^{17}$ Kitcher erläutert dies wie folgt: „functional analyses“ ,[...] provide a functional specification of the kind of processing, or faculty, required for a given cognitive task; alternatively, they decompose a cognitive task into its basic subtasks and so reveal that it involves elements that cannot be supplied by the senses“. ${ }^{18}$ Bezogen auf die Diskursivitätsthese heißt das, dass die Einheit des Bewusstseins durch die Analyse der konzeptuellen Handlungen des Verstandes erklärt wird. Die Diskursivität menschlicher Begriffe bzw. des menschlichen Verstandes liefert dabei die Begründung, warum Einheit und damit Identität diejenigen diskursivlogischen Eigenschaften sind, durch die sich menschliches Bewusstsein transzendentalphilosophisch auszeichnet. Zumindest in dieser Hinsicht scheint eine funktionalistische Vermögensanalyse vielversprechend $\mathrm{zu}$ sein, auch wenn damit nicht entschieden ist, ob der Funktionalismus überhaupt mit dem transzendentalphilosophischen Projekt vereinbart werden kann.

\section{Literaturverzeichnis}

Allison, Henry E., 1996, Idealism and Freedom. Essays on Kant's Theoretical and Practical Philosophy, Cambridge: Cambridge University Press.

Allison, Henry E., 2004, Kant's Transcendental Idealism, An Interpretation and Defense, New Haven / London: Yale University Press.

Baum, Manfred, 2002, „Logisches und personales Ich bei Kant“, in: Dietmar H. Heidemann (Hrsg.): Probleme der Subjektivität in Geschichte und Gegenwart, Stuttgart-Bad Cannstatt: frommann-holzboog, 107-123.

Brook, Andrew, 1994, Kant and the Mind, Cambridge: Cambridge University Press.

Düsing, Klaus, 2004, "Spontane, diskursive Synthesis. Kants neue Theorie des Denkens in der kritischen Philosophie”, in: Sabine Doyé u. a. (Hrsg.), Metaphysik und Kritik. Festschrift für Manfred Baum zum 65. Geburtstag, Berlin/New York: De Gruyter, 83-107.

\footnotetext{
${ }^{17}$ Vgl. Brook 1994: 115, auch 152, 170, 194, 224.

${ }^{18}$ Vgl. Kitcher 1990: 20f., ebenso 12, 111, 207.
} 
Heidemann, Dietmar H., 2013, „Kant and Non-Conceptual Content: The Origin of the Problem“, in: Dietmar H. Heidemann (ed.), Kant and Non-Conceptual Content, Abindon: Routledge, 1-10.

Heidemann, Dietmar H., 2004, „Kants Grammatik des Verstandes. Erkenntnistheoretische Untersuchungen zum Zusammenhang von Urteil und Kategorie“, in: Dieter Hüning u.a. (Hrsg.): Aufklärung durch Kritik, Berlin: Dunker \& Humblot, 189-218.

Heidemann, Dietmar H., 2002, „Anschauung und Begriff. Ein Begründungsversuch des Stämmedualismus in Kants Erkenntnistheorie“, in: Kristina Engelhard (Hrsg.): Aufklärungen. Festschrift für Klaus Düsing zum 60. Geburtstag, Berlin: Dunker \& Humblot, 65-90.

Kants Gesammelte Schriften, Akademie der Wissenschaften (vormals: Preußische Akademie der Wissenschaften), Berlin: Walter de Gruyter 1900ff.

Kant, Immanuel, Kritik der reinen Vernunft, Jens Timmermann (Hrsg.), Hamburg: Meiner 1998 (A für die erster Auflage, B für die zweite Auflage).

Kitcher, Patrica, 1999, „Kant on Selfconsciousness“, in: Philosophical Review, 108, 345-386.

Kitcher, Patrica, 1990, Kant's Transcendental Psychology, New York: Oxford University Press

Locke, John, An Essay concerning Human Understanding, P. H. Nidditch (Hrsg.), Oxford 1975.

Thiel, Udo, 2015, „Unities of the Self from Kant to Locke“, in: Kant Yearbook, 7, $139-165$ 
Vanzo, Alberto, 2012, Kant e la formazione die concetti, Trento: Pubblicazione di Vrifiche 48. 